\title{
Estratégias operacionais visando a sustentabilidade hídrica e energética de simulação e dimensionamento de redes de abastecimento de água
}

\author{
Operational strategies aiming at the water and energy sustainability of simulation and sizing of
} water supply networks

Estrategias operativas que apuntan a la sostenibilidad del agua y la energía de la simulación y el dimensionamiento de las redes de suministro de agua

\section{Resumo}

A preocupação com sustentabilidade aplica-se as redes de distribuição hidráulicas, sendo responsáveis pelo transporte de um bem cada vez mais precioso que é a água potável. Estes sistemas tem em média perdas elevadas por diversos motivos. O foco aqui é a questão do excesso de pressão na rede ocasionado pela operação ineficaz. Assim, o investimento em melhorias dos sistemas torna-se essencial. Deste modo, este trabalho objetiva a proposição de procedimento para estratégias operacionais para controle de pressões para redução de perdas de água e análise financeira paralela. A metodologia é baseada em simulações hidráulicas de sistema real de área com topográfica acidentada estabelecendo-se cenários de análise e fazendo análises econômicas. O estudo foi feito para uma rede de distribuição real. Os resultados demostraram que ocorreram melhorias no controle de pressões e consequente redução de perdas de água nos cenários com atribuição de válvulas a trechos, proposição de bombas, variação de níveis de reservatórios entre outras proposições. Concluiu-se que o procedimento proposto, embora baseado em simulações computacionais pode ser considerado importante, pois a experiência foi feita para um sistema real que representa um conjunto expressivo de sistemas encontrados em operação.

Palavras-chave: Análise dos resultados; Custos financeiros; Simulações computacionais.

\begin{abstract}
The concern with sustainability applies to water distribution networks, being responsible for transporting an increasingly valuable asset that is drinking water. These systems have on average high losses for various reasons. The focus here is the issue of over-pressure in the network caused by inefficient operation. Thus, investing in system improvements becomes essential. Thus, this work aims at proposing a procedure for operational strategies to control pressures to reduce water losses and parallel financial analysis. The methodology is based on hydraulic simulations of real area system with rugged topography establishing analysis scenarios and making economic analyzes. The study was done for an actual distribution network. The results showed that there were improvements in the control of pressures and consequent reduction of water losses in the scenarios with attribution of valves to sections, proposition of pumps, variation of levels of reservoirs among other propositions. It was concluded that the proposed procedure, although based on computational simulations can be considered important, since the experiment was done for a real system that represents an expressive set of systems found in operation.
\end{abstract}

Keywords: Analysis of results; Computational simulations; Financial costs. 


\begin{abstract}
Resumen
La preocupación por la sostenibilidad se aplica a las redes de distribución de agua, siendo las encargadas de transportar un bien cada vez más valioso que es el agua potable. Estos sistemas tienen en promedio altas pérdidas por varias razones. El foco aquí es el problema de la sobrepresión en la red causada por un funcionamiento ineficiente. Por lo tanto, invertir en mejoras del sistema se vuelve esencial. Así, este trabajo tiene como objetivo proponer un procedimiento de estrategias operativas para controlar las presiones para reducir las pérdidas de agua y un análisis financiero paralelo. La metodología se basa en simulaciones hidráulicas de sistema de área real con topografía accidentada estableciendo escenarios de análisis y realizando análisis económicos. El estudio se realizó para una red de distribución real. Los resultados mostraron que hubo mejoras en el control de presiones y consecuente reducción de pérdidas de agua en los escenarios con atribución de válvulas a tramos, proposición de bombas, variación de niveles de reservorios entre otras proposiciones. Se concluyó que el procedimiento propuesto, aunque basado en simulaciones computacionales, puede considerarse importante, ya que el experimento se realizó para un sistema real que representa un expresivo conjunto de sistemas encontrados en operación.
\end{abstract}

Palabras clave: Análisis de resultados; Costos financieros; Simulaciones computacionales.

\title{
1. Introdução
}

A gestão eficiente dos recursos hídricos é essencial para a conservação da água, destacando as redes de distribuição que podem atingir perdas na ordem de até $40 \%$. Vieira (2013) trata alguns fatores que podem contribuir para a falta de eficiência de um sistema de distribuição para abastecimento, entre eles destacam-se a falta de investimentos no setor. Outro fator que contribui é a falta de medidores e de qualificação profissional, para o sistema de abastecimento de água que gera um desperdício deste bem tão valioso na captação armazenamento e distribuição gerando grandes prejuízo (Reis, 2017).

Segundo Kumar et al. (2014) o EPANET é um software que executa a simulação de um período prolongado de um comportamento hidráulico e da qualidade da água em redes de tubulação pressurizada. O EPANET monitora o fluxo de água em cada tubo, a pressão em cada nó, a altura da água em cada tanque e a concentração de uma espécie química em toda a rede durante um período de simulação (Nallanathel, et al., 2018).

Tack et al. (2014) descrevem o programa EPANET como sendo um software livre utilizado para realizar simulações hidráulicas de sistemas de água, as simulações efetuadas pelo EPANET geram respostas importantes tais como, vazão e pressão na rede, velocidade, perda de carga, entre outros. Ramana et al. (2015) descreve que a ferramenta EPANET é eficiente para redes de distribuição de água, com uma ampla gama de aplicações não havendo limites para expansão. O software EPANET pode ser usado para muitos tipos diferentes de análise hidráulica da rede de distribuição.

Maruf et al. (2015) trata os dispositivos para representar a configuração do sistema de distribuição onde são fornecidas informações detalhadas sobre o tubo como diâmetro, comprimento, nós e válvulas, desta forma as pressões em todas as junções e os fluxos com suas velocidades em todos os tubos são viáveis o suficiente para fornecer água adequada para a rede.

Segundo Uber et al. (2018) os modelos de simulação de infraestrutura de água, com o EPANET, têm desempenhado um papel importante no desenvolvimento de tecnologias de simulação para os sistemas de distribuição de água pluviais e esgotos sanitários. Esse software tem várias funções para os sistemas de infraestrutura, municípios, hidrelétricas e consultores usam esse pacote para garantir serviços adequados de água e para proteger a saúde pública.

Antonowicz et al. (2018) desenvolve um método de utilização do solver EPANET para realizar simulações, e com isso consegue escolher a melhor operação do cenário da rede de abastecimento de água de acordo com um critério especificado. Foi para este propósito, que o aplicativo baseado na web foi desenvolvido. O aplicativo executa simulações simples e cíclicas com a etapa especificada de alteração dos valores das variáveis de processo selecionadas como desempenho da bomba e taxa de abertura da válvula.

A realização do estudo sobre a ferramenta de domínio desenvolvida no trabalho de Suse et al. (2014) EPANET-MSX (EPANET Multi-Species Extension, 2008), utiliza a análise de casos hipotéticos que permitiram avaliar as possibilidades de 
simulação, concluí que trabalho a utilização desta ferramenta em problemas reais poderá demonstrar operações da rede hidráulica para melhoria da qualidade de água, evitando descargas de grandes volumes de água para esta finalidade circulação de "zonas mortas" ou com baixas velocidades.

O procedimento proposto de simulações hidráulicas por Farina et al. (2014) envolve a aplicação de simulação de forma iterativa, introduzindo uma correção adequada da rugosidade do tubo em cada iteração através do modelo EPANET para representar perdas de carga precisas nos tubos levando em consideração as posições reais dos usuários ao logo da tubulação. Em dois estudos de caso da cidade de Gênova, mostrou que a nova abordagem é mais precisa em redes ramificadas com grande tubo, que nos casos de uma descrição muito detalhada do sistema de rede de tubulação, o uso de demanda tradicional ou real produz quase os mesmos resultados.

Um dos métodos apresentado por Saqib (2017) trata de nova abordagem multimodal e multi-escala para detecção e localização de vazamentos em redes de tubulações de água com sensores de vibração, são usados para localizar o vazamento dentro deste segmento. Para validar a eficácia da abordagem que necessita de dados de pressão e vibração utiliza-se o software EPANET que inclui vazão e pressão em vários pontos da tubulação, em seguida integrou o modelo de vibração no MATLAB, já que a EPANET não inclui modelos para medições de vibração. Os resultados extensivos da simulação mostram a eficácia do esquema proposto no fornecimento de detecção e localização precisas de vazamentos.

No estudo de Mohammad et al. (2015), foi analisado a área de Banani, uma rede de distribuição da capital de Bangladesh, Daca, foi utilizada um arquivo AutoCAD, que foi exportado e modelada usando o EPANET, o valor do modelo (DWAAS) Water Supply \& Sewerage Authority (DWASA) são significativamente semelhantes. A verificação cruzada foi feita para garantir sua precisão e os erros foram resolvidos. O sistema de distribuição existente na área de Banani é capaz de suprir a demanda média diária e a demanda diária máxima. Assim, o modelo foi concluído e, após a calibração necessária, os resultados foram gerados.

O modelo hidráulico empregado por Goulart (2015) e Santos (2016) desenvolve o aprimoramento de iterações do método de calibração de redes de distribuição de água proposto por Silva (2003) trata-se implementação técnica de algoritmo genético, calibrando duas redes de distribuição de água reais da cidade de São Carlos (SP), e ajustando parâmetros, de rugosidades e de vazamentos.

\section{Metodologia}

O procedimento metodológico o para melhoria de operação eficiente proposto é apresentado na Figura 1. 
Figura 1 - Procedimento proposto para eficiência de operação de redes de redução de perdas de água.

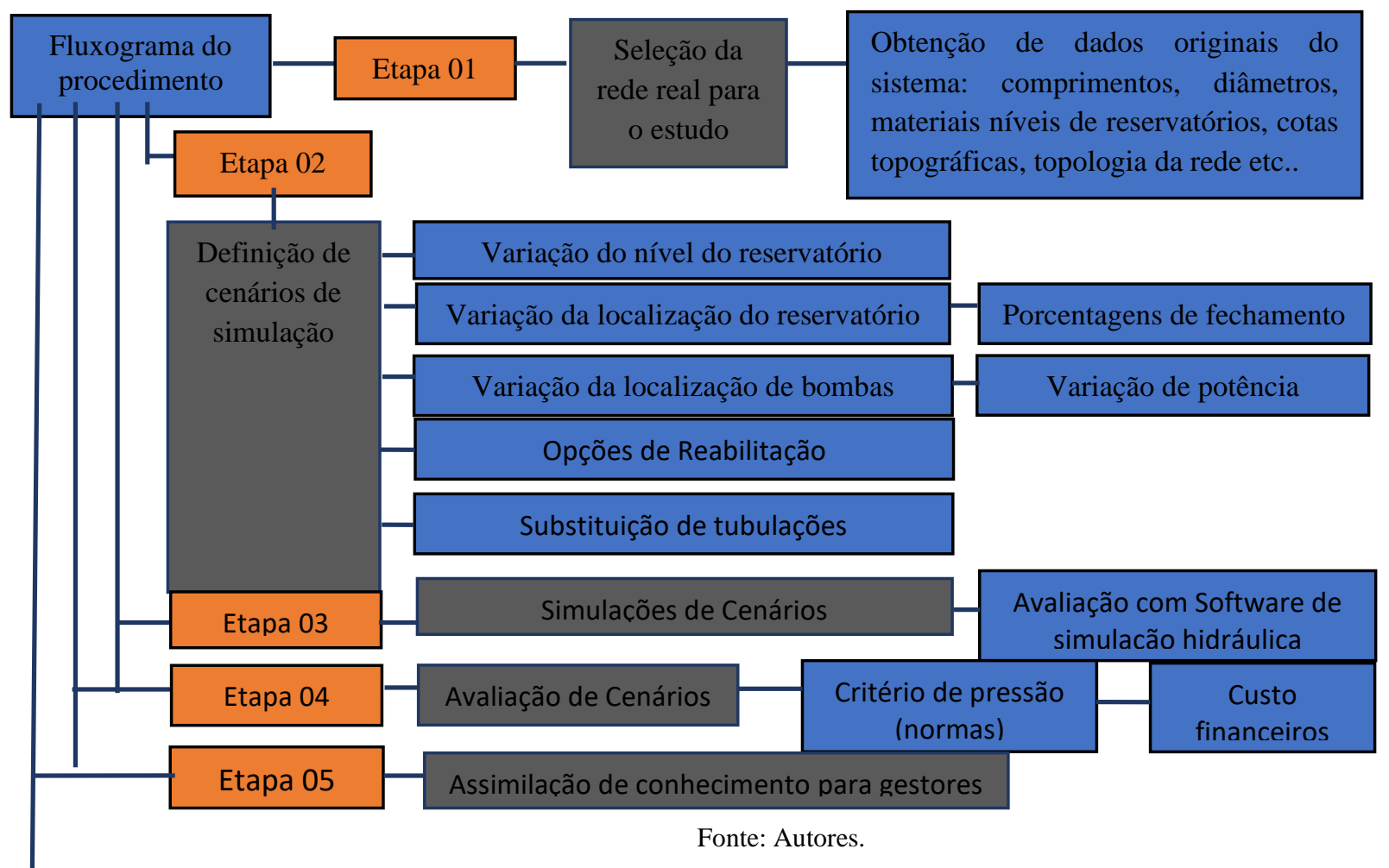

- ETAPA 1: Escolha de rede real para o estudo - Nesta etapa é escolhida rede real para aplicação onde poderão ser obtidas informações tais como: comprimentos da rede, diâmetros das tubulações, materiais, cotas topográficas, níveis de reservatórios, localização de dispositivos hidráulicos no sistema. Tal etapa é importante para garantir a confiabilidade das informações coletadas em campo necessárias para construção básica de rede. O setor eleito foi selecionado por ser isolado, pois fica garantido o balanço de variáveis de controle;

- ETAPA 2: Definição de Cenários de simulação - Os cenários foram, principalmente, baseados em: Alteração de níveis de reservatórios; inserção de válvulas redutoras de pressão no sistema; inserção de bombas no sistema; alterações das tubulações e Reabilitação das tubulações.

Foram verificadas nesta fase as possibilidades de alterações de parâmetros hidráulicos e localização de acessórios da rede e procedimentos na rede de distribuição de água de modo a possibilitar que uma situação hidráulica de melhor eficiência e consequente melhor custo se estabeleça, as simulações básicas feitas em condições natural hidráulica demostra-se faixa de pressões acima do que recomenda a norma (Associação Brasileira de Normas Técnicas, 2017), provocando grandes vazamentos e baixa eficiência hídrica e energética.

Esta etapa é de grande importância, pois a definição dos cenários possibilita abordar todas as possíveis alterações do sistema que poderão ser encontradas em sistemas típicos constituindo basicamente: distribuição de bombas e respectivas potências no sistema, válvulas com porcentagens de fechamentos, variação dos níveis reservatórios, variações de rugosidades e diâmetros das tubulações.

- ETAPA 3: Simulação de cenários - Nesta etapa com o uso do software Epanet de simulação são geradas as situações hidráulicas originadas a partir dos cenários estabelecidos na etapa anterior. O programa realiza simulações estática e dinâmica do comportamento hidráulico da rede pressurizadas permitindo a visualização dos valores de pressão em cada trecho, as pressões nos nós e o nível de água nos reservatórios; 
- ETAPA 4: Avaliação de cenários. A avaliação dos cenários foi feita em termos principalmente de variações de pressões nas redes que podem ser associadas a custos e a ligação pode ser feita com perdas de água;

- ETAPA 5: Assimilação de conhecimentos para gestores - Com os resultados obtidos a partir da rede real e cenários comuns em redes de água brasileiras, gerou-se conhecimentos que podem ser passados a gestores para auxiliar na tomada decisões, respeitados os diferenciais de situações analisadas.

\section{Resultados e Discussão}

\subsection{Cenários de análise}

Os estudos foram conduzidos por análises de cenários desenvolvidos a partir do sistema original, com situações hidráulicas possíveis de ocorrer em sistemas reais conforme descrição na metodologia. Na Figura 2 (a) e (b) respectivamente são representadas as situações de uma rede real em condições de monitoramento originais e com a instalação de dispositivos hidráulicos como bombas e válvulas.

Figura 2 - (a) Rede original com o RNF= 957 metros (b) Instalação de válvulas e bombas.
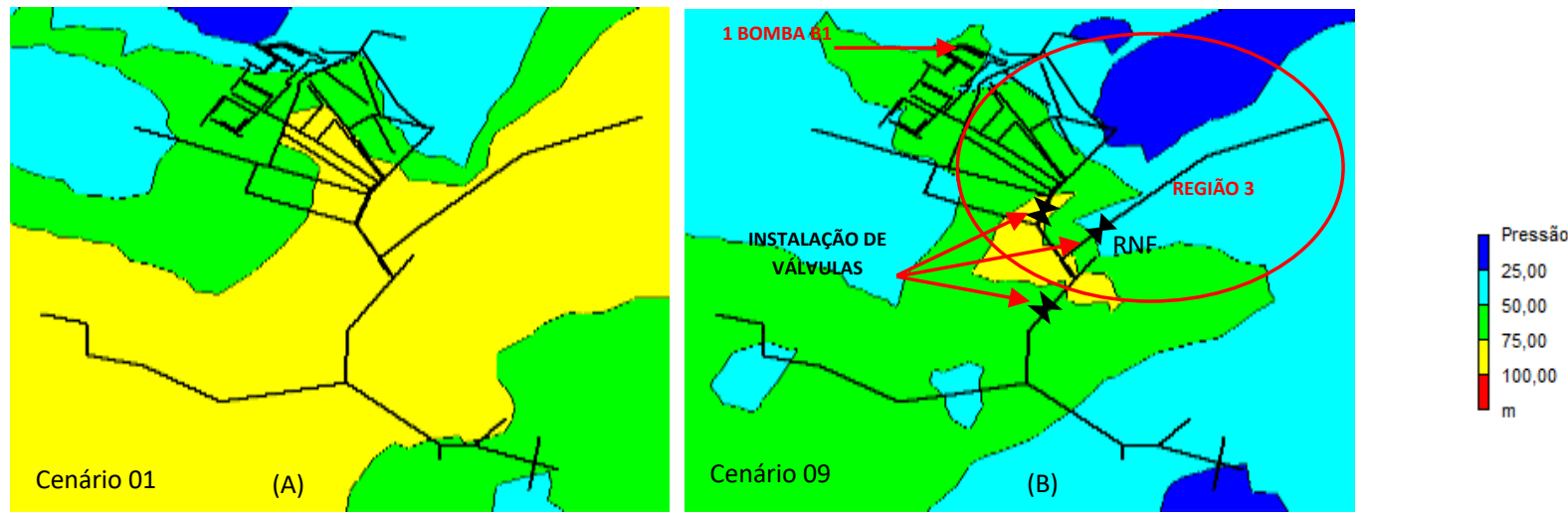

Fonte: Autores.

Na Figura 3 verifica-se uma comparação do cenário 09 com o cenário 01 em termos de pressão, a inserção de três válvula, associado a bomba resulta em uma alteração de pressão. Observa-se que as pressões nos nós estão fora do que recomenda a (Associação Brasileira de Normas Técnicas, 2017) valores entre 10 a 50 mca. Em todo o comportamento das pressões distribuídas na rede em estudo apenas $26,72 \%$ dos nós atenderam a recomendação da a norma (Associação Brasileira de Normas Técnicas, 2017). Com este cenário observa-se ajuste de pressões em alguns pontos principalmente dos nós 1 a 16. 
Figura 3 - comparação de pressão entre cenário 01 e 09 com inserção de bomba.

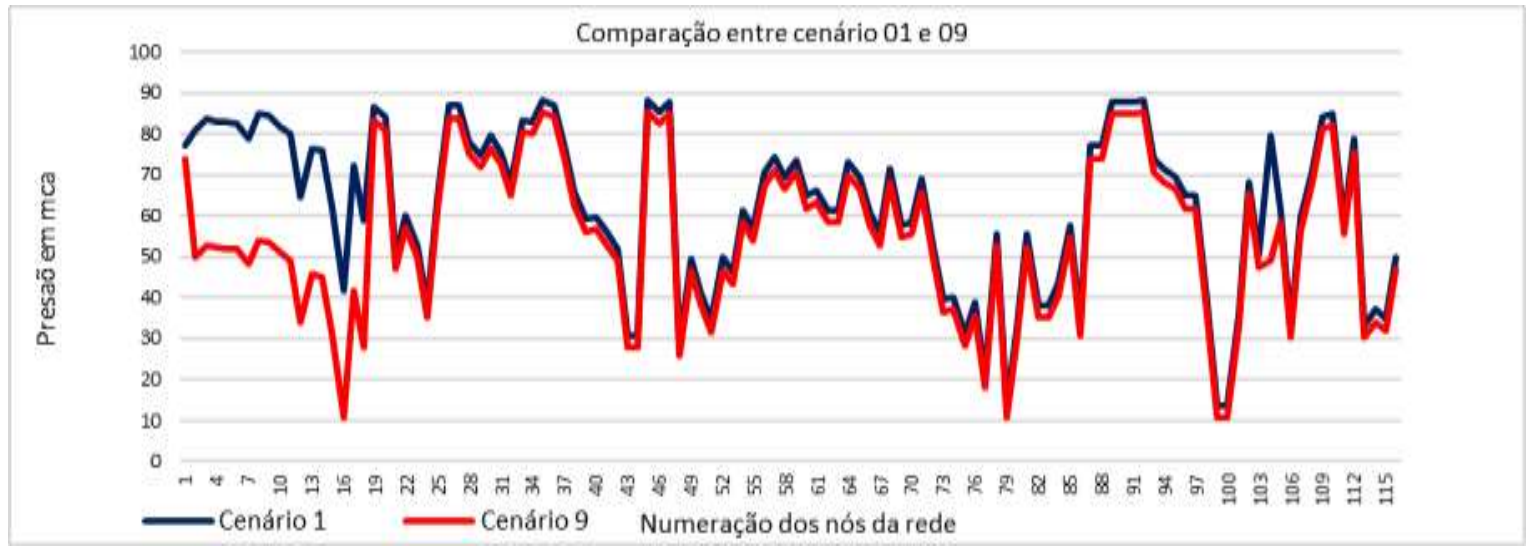

Fonte: Autores.

Na Figura 4 (b), verifica-se uma comparação do cenário 32 com o cenário 01 a inserção de quatro válvulas, associado a bomba que resulta em uma alteração de pressão. O cenário 32 é o resultado de uma inserção de bomba na região com maiores altitudes entre o trecho 96 com o reservatório a 944 metros, reforçando a pressão nos pontos mais altos que eventualmente ficam mais despressurizadas. Observa-se um grande aumento das regiões de faixas de pressão entre 25 e 50 mca e entre 0 e 25 mca.

Na Figura 4 (c) verifica-se uma comparação do cenário 33 com o cenário 01 a inserção de quatro válvulas, associados as duas bombas que resulta em uma alteração de pressão, diminuindo a média das pressões entre 75 a 100 m.c.a. O cenário 33 é o resultado de duas inserções de bombas nas regiões com maiores altitudes entre no trecho 96 e outra bomba no trecho 13 com o reservatório a 944 metros, reforçando a pressão nos pontos mais altos que eventualmente ficam mais despressurizadas. Comparando este cenário com o cenário 32 observa-se que os valores de pressões entre 50 e 75 mca tendem a ficar mais próximos dos valores da norma.

Figura 4 - (a) Rede original com o RNF= 957 metros (b) Cenário 32 (b) Cenário 33.
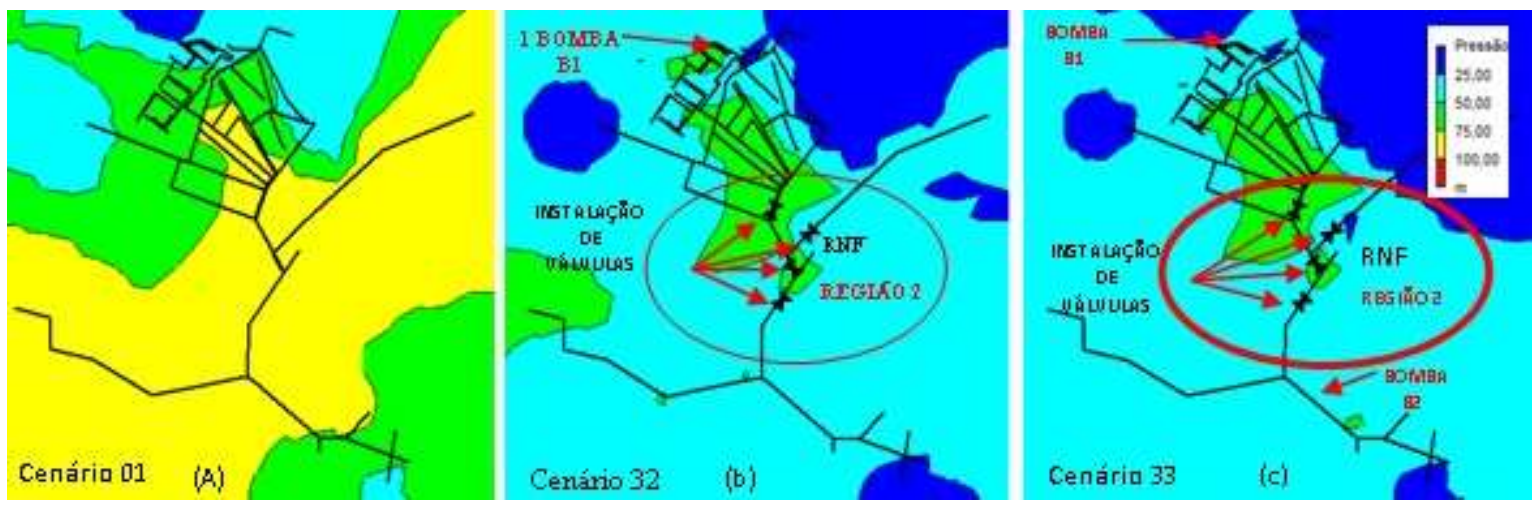

Fonte: Autores.

Na Figura 5 é demostrado um gráfico comparativo de pressões entre os cenários 32 e 01, este comparativo é atribuído com o acréscimo de uma bomba com atribuição de quatro válvula e com o ajuste da cota do reservatório. Observa-se que os valores de pressão são rebaixados de maneira acentuada com relação aos nós, ficando mais próximos possíveis dos valores recomendados por norma com relação a comparação anterior. 
Figura 5 - Comparação de pressão entre cenário 01 e 32 com inserção de bomba.

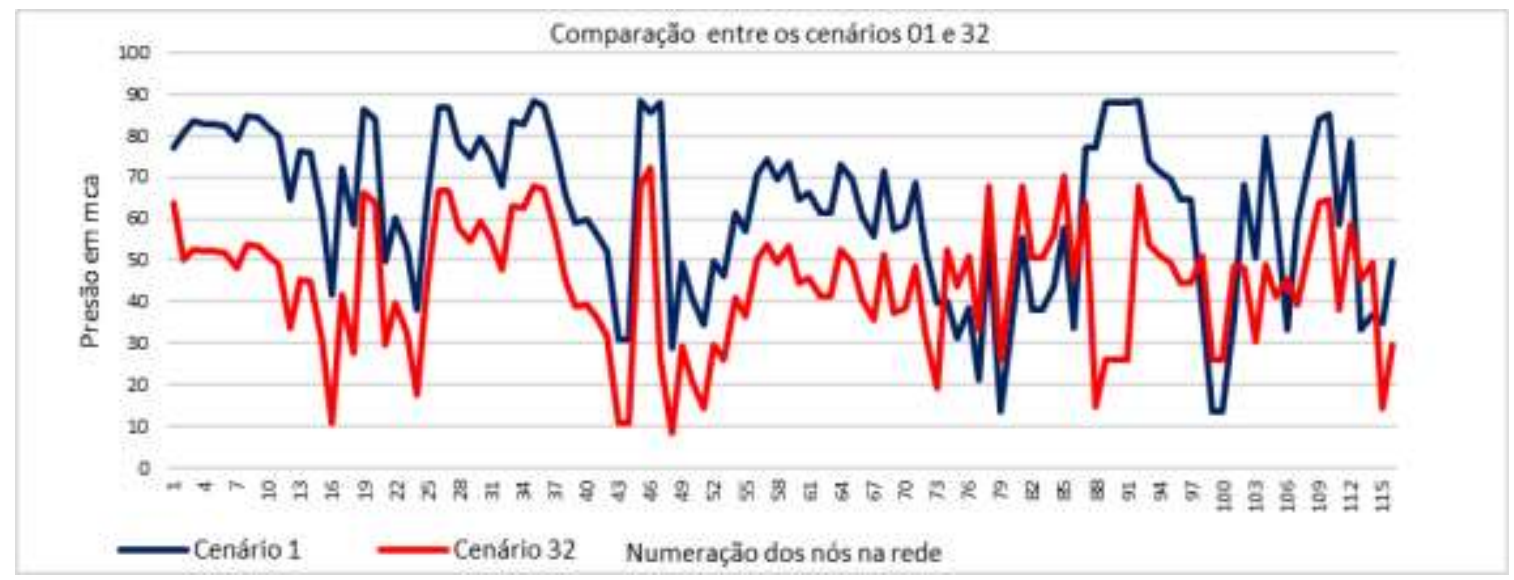

Fonte: Autores.

Na Figura 6 é demostrado um gráfico comparativo de pressões entre os cenários 33 e 01, este comparativo é atribuído com o acréscimo de duas bombas com atribuição de quatro válvulas e com o ajuste da cota do reservatório, diminuindo a pressão. Observa-se a queda da linha vermelha mais acentuada ainda com relação ao cenário 32 com os valores ainda mais próximos dos valores previstos em norma.

Figura 6 - Comparação de pressão entre cenário 01 e 33 com inserção de bomba.

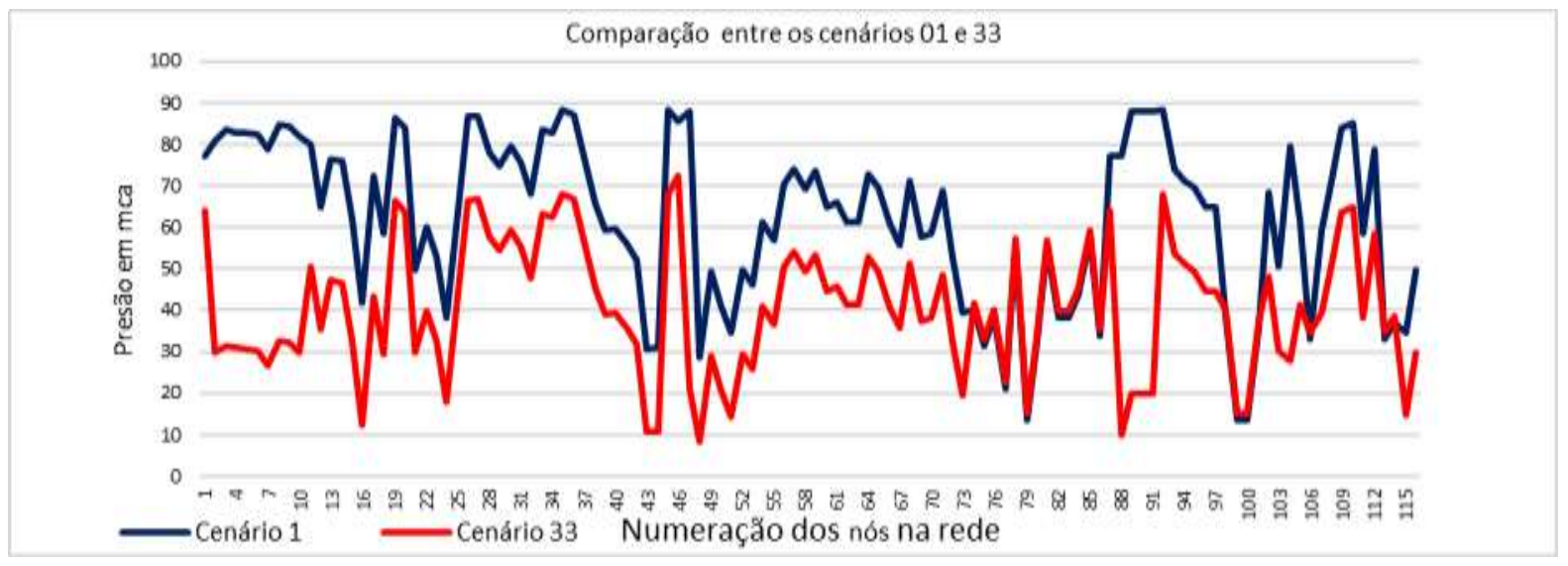

Fonte: Autores.

Na Figura (7b), verifica-se uma comparação do cenário 34 com o cenário 01 a inserção de três válvulas, associado as duas bombas que resulta em uma alteração de pressão, que destaca uma região 1 diminuindo a média das pressões entre 75 a 100 m.c.a. O cenário 34 é o resultado de duas inserções de bombas na região com maiores altitudes uma entre o trecho 96 e outra bomba ao nó trecho 13 com o RNF= 940 metros, reforçando a pressão nos pontos mais altos que eventualmente ficam mais despressurizadas, e com três válvulas de controle em trechos diferentes.

Na Figura (7c), verifica-se uma comparação do cenário 35 com o cenário 01 a inserção de quatros válvulas, associado a uma bomba que resulta em uma alteração de pressão. Destacando uma região 1 diminuindo a média das pressões entre 75 a 100 m.c.a. Os cenários 35 é o resultado da inserção de bomba na região com maiores altitudes entre o nó trecho 96 e com o $\mathrm{RNF}=940$ metros. Comparando os dois cenários, verifica-se boas melhorias de pressão no ponto mais alto, mas ainda existem pontos com excesso de pressão.

Figura 7 - (a) Rede original com o RNF= 957 metros (b) Cenário 34 (c) Cenário 35. 


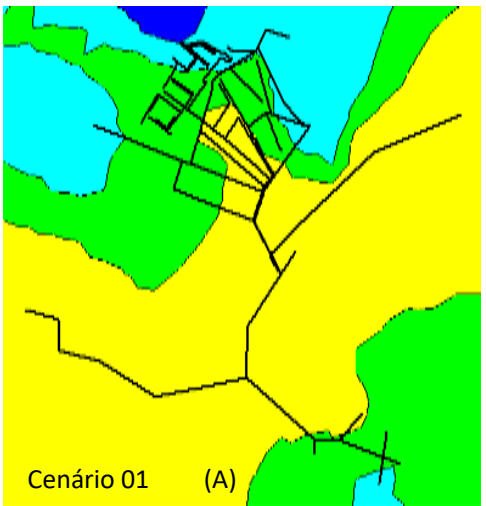

Cenário 01
(A)

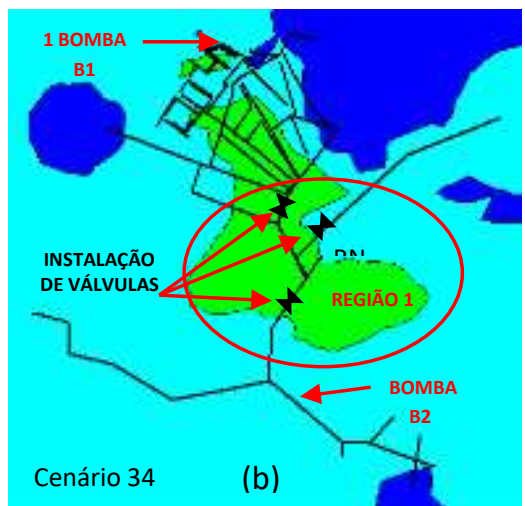

Fonte: Autores.

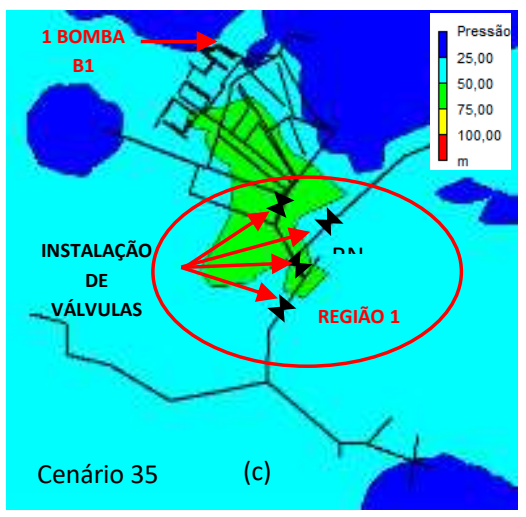

(c)

Na Figura 8 é demostrado um gráfico comparativo de pressões entre os cenários 34 e 01, este comparativo é atribuído com o acréscimo de duas bombas com atribuição de quatro válvulas e com o ajuste da cota do reservatório, diminuído a pressão em grandes concentrações. Observa-se uma redução em linhas gerais da linha vermelha de faixas de pressões com relação a linha azul, entretanto não tão próximo da norma como a situação 33.

Figura 8 - Comparação de pressão entre cenário 01 e 34 com inserção de bomba.

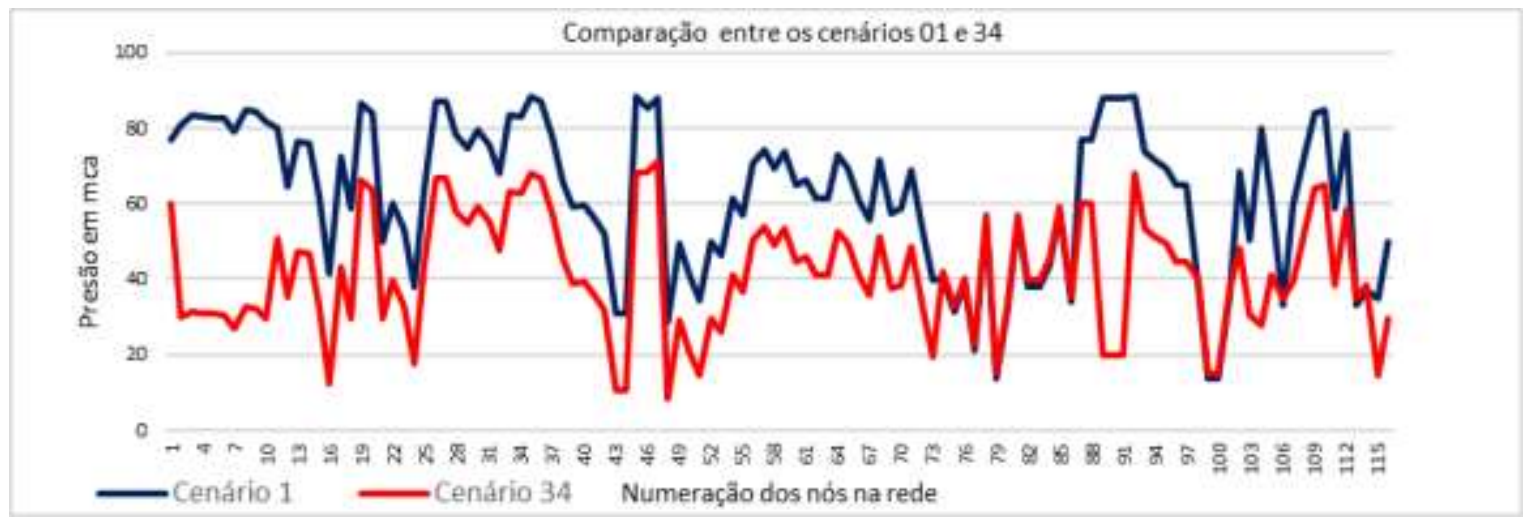

Fonte: Autores.

Na Figura 9 é demostrado um gráfico comparativo de pressões entre os cenários 35 e 01, este comparativo é atribuído com o acréscimo de uma bomba com atribuição de quatro válvula e com o ajuste da cota do reservatório, diminuído a pressão em grandes concentrações. As diferenças entre valores pressões ajustadas para o cenário 35 ficaram próximas a do cenário 33, diferenciando os dispositivos previstos. 
Figura 9 - Comparação de pressão entre cenário 01 e 35 com inserção de bomba.

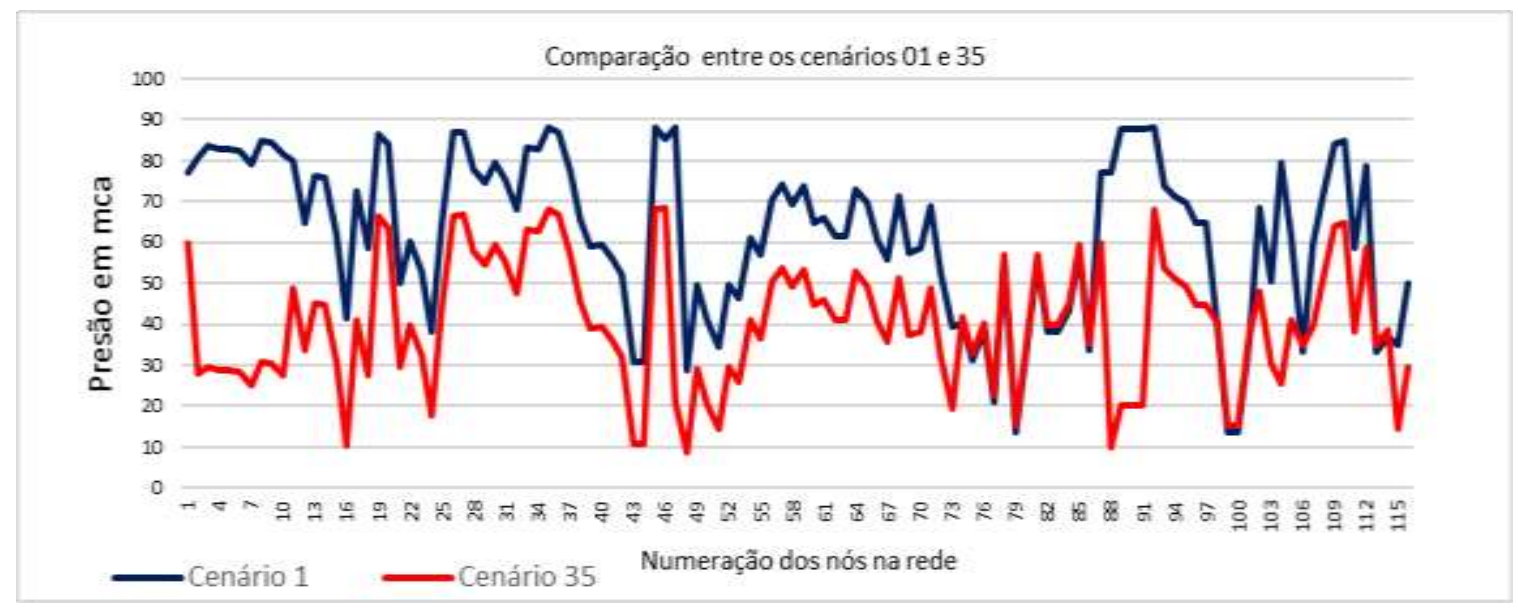

Fonte: Autores.

Na Figura 10 é demostrado um grafíco comparativo de pressões entre os cenário 35 e e o mesmo cenário que utilizou a tecnica de reabilitação, este comparativo é atribuido ao gráfico com a alteraçãoes da rugosidade de 4 trechos que resultou na diminuição pressão em boa parte da rede.

Figura 10 - Gráfico comparativo de pressão entre cenário 35 utilizando técnica de reabilitação da rede.

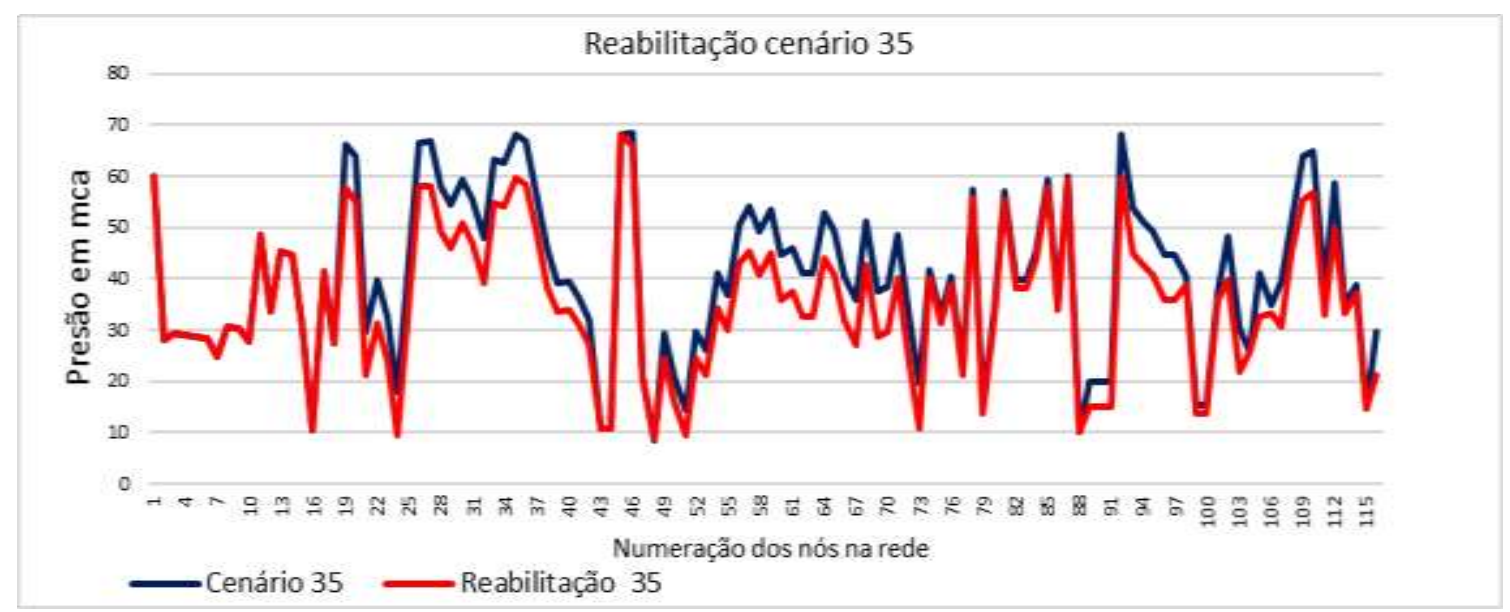

Fonte: Autores.

A técnica de reabilitação no cenário 35 teve um atendimento de 80,17\% em relação ao cenário 35 que teve atendimento de $70,69 \%$, registrando uma melhoria de 9,48 p.p. (pontos percentuais).

\subsection{Análise de custo de investimentos e análises de melhoria de pressão}

A Figura 11 trata das relações dos investimentos em reais envolvidos na implantação das possíveis soluções com introdução de dispositivos hidráulicos em relação a porcentagem de todos os nós da rede em que são atendidas as pressões normatizadas. A variação de pressão ocorreu dentro da norma (Associação Brasileira de Normas Técnicas, 2017). Dentre todos os inúmeros cenários analisados selecionou-se os cenários: 09, 10, 32, 33, 34 e 35. Os custos que subsidiaram o gráfico foram levantados com base nos dados fornecidos pelo Sistema Nacional de Pesquisa de Custos e Índices da Construção Civil (SINAPI, 2019). 
Figura 11 - Investimento para melhoria de rede de distribuição com relação a cada cenário.

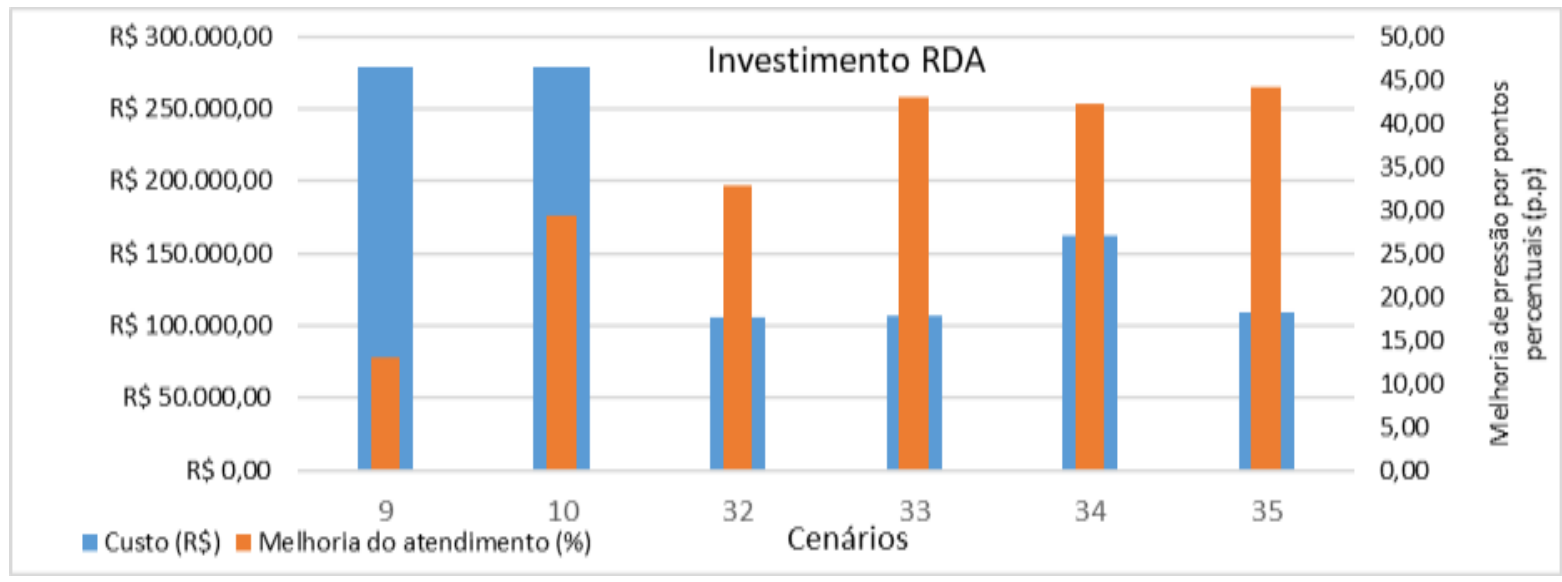

Fonte: Autores.

A relação do comportamento no gráfico demonstrado na Figura 11 oferece uma visão nos estudos com um comparativo de pressões em determinados cenários, esse possível método ajuda a verificar o melhor custo atribuído em investimentos de rede de distribuição. As características necessárias para a determinação dos custos de implantação de uma rede de distribuição de água envolvem os serviços e os custos de material, foram abordadas.

Observa-se que os cenários 32, 33 e 35 apresentam os melhores resultados com relação em termos de custos financeiros representando os custos mínimos. Já os cenários 33, 34 e 35 podem ser considerados os mais eficientes em termos de valores máximos de porcentagens de pontos com pressões médias atendidas para os padrões de normas (Associação Brasileira de Normas Técnicas, 2017). O cenário 35 trata-se da melhor solução contemplando os dois objetivos. Este cenário representa a inserção de quatro válvulas uma no trecho 1 com diâmetro de $100 \mathrm{~mm}$ e parâmetro de controle de 50 m.c.a outra no trecho 65 com diâmetro de $200 \mathrm{~mm}$ e parâmetro de controle de 26 m.c.a e outra que liga o trecho 62 com diâmetro de 150 mm e parâmetro de controle de 76 m.c.a, a quarta válvula no trecho 113 com diâmetro de $50 \mathrm{~mm}$ e parâmetro de controle de 25 m.c.a. e com ajuste do nível do reservatório de 940 metros com a inserção de duas bombas uma no trecho 96 e outra no trecho 13 as duas bombas com as mesmas características de $3 \mathrm{cv}, 3500$ r.p.m, trifásica.

No cenário 35, a Tabela 1 compôs-se os custos de implantação para diâmetros nominais de 04 válvulas (DN) de 50, 100, 150 e 200, considerando-se para implantação duas bombas de 3,5 cv e a modificação do nível do reservatório. Mantendo o trecho que liga o nó 43 ao nó 44 e o trecho que liga o reservatório ao nó 1. No cenário 35 teve atendimento de 70,96 \% em relação ao cenário 01 que teve atendimento de 26,72 \%, registrando uma melhoria de 44,24 p.p (pontos percentuais). 
Tabela 1 - Relações dos investimentos atribuídas ao cenário 35.

\begin{tabular}{|c|c|c|c|c|c|c|}
\hline Trecho & $\begin{array}{c}\text { Cenário } \\
35\end{array}$ & $\begin{array}{c}\text { Mudança de } \\
\text { Diâmetro }\end{array}$ & $\begin{array}{c}\text { Comprimento } \\
\mathbf{m}\end{array}$ & $\begin{array}{c}\text { preço } \\
\text { médio (R\$) }\end{array}$ & $\begin{array}{l}\text { Escavação } \\
\text { e reaterro }\end{array}$ & Total (R\$) \\
\hline Trecho 2 & DN 150 & DN 50 & 9,10 & 1,72 & 45 & $\mathrm{R} \$ 704,34$ \\
\hline Trecho 63 & DN 150 & DN 75 & 220,40 & 2,3 & 45 & $\mathrm{R} \$ 22.811,40$ \\
\hline Trecho 53 & DN 75 & DN 50 & 16,00 & 1,72 & 45 & $\mathrm{R} \$ 1.238,40$ \\
\hline Trecho 54 & DN 75 & DN 50 & 45,00 & 1,72 & 45 & $R \$ 3.483,00$ \\
\hline Trecho 51 & DN 75 & DN 50 & 52,00 & 1,72 & 45 & $\mathrm{R} \$ 4.024,80$ \\
\hline Trecho 58 & DN 75 & DN 50 & 182,30 & 1,72 & 45 & $\mathrm{R} \$ 14.110,02$ \\
\hline Trecho 59 & DN 100 & DN200 & 22,40 & 4,02 & 45 & $\mathrm{R} \$ 4.052,16$ \\
\hline Trecho 69 & DN 100 & DN 50 & 271,25 & 1,72 & 45 & $\mathrm{R} \$ 20.994,75$ \\
\hline Trecho 72 & DN 100 & DN 50 & 136,20 & 1,72 & 45 & $\mathrm{R} \$ 10.541,88$ \\
\hline Trecho 74 & DN75 & DN 50 & 72,90 & 1,72 & 45 & $R \$ 5.642,46$ \\
\hline Trecho 39 & DN 75 & DN 50 & 178,12 & 1,72 & 45 & $\mathrm{R} \$ 13.786,49$ \\
\hline Trecho 96 & \multicolumn{2}{|c|}{ bomba $3,5 \mathrm{cv}$} & & 1200 & 191,32 & $\mathrm{R} \$ 1.391,32$ \\
\hline Trecho 13 & \multicolumn{2}{|c|}{ bomba $3,5 \mathrm{cv}$} & & 1200 & 191,32 & $\mathrm{R} \$ 1.391,32$ \\
\hline $\begin{array}{c}\text { Reservatório } \\
\text { elevado }\end{array}$ & & & & $3.647,47$ & & $R \$ 3.647,47$ \\
\hline válvulas & DN 50 & DN 50 & & 104,72 & & $\mathrm{R} \$ 104,72$ \\
\hline válvulas & DN 100 & DN 100 & & 333,3 & & $\mathrm{R} \$ 333,30$ \\
\hline válvulas & DN 150 & DN 150 & & 443,91 & & $\mathrm{R} \$ 443,91$ \\
\hline \multirow[t]{2}{*}{ válvulas } & DN 200 & DN 200 & & 517,9 & & $\mathrm{R} \$ 517,90$ \\
\hline & Anterior & Atual & & Melhoria & & Total \\
\hline $\begin{array}{l}\text { Melhoria } \\
\text { pressão }\end{array}$ & $26,72 \%$ & $70,96 \%$ & & 44,24 & p.p. & $\begin{array}{c}\mathrm{R} \$ \\
109.219,64\end{array}$ \\
\hline
\end{tabular}

Fonte: Autores.

\section{Conclusão}

No estudo de análises de pressões de maneira estratégica através da inserção de equipamentos apropriados ao sistema de distribuição, desta forma foi possível atribuir, com a ferramenta EPANET, diferentes cenários de simulação hidráulica de modo a diminuir as principais perdas na distribuição. Os parâmetros utilizados na avaliação nas melhores soluções foram os custos dos dispositivos hidráulicos no sistema e o atendimento da maior quantidade possível de pressões de nós da rede.

Mostrou-se necessário a combinação de dispositivos hidromecânicos como bombas, válvulas e a alteração do nível do reservatório em diferentes condições para chegar a bons resultados. Na análise global definiu-se que o cenário 35 conseguiu contemplar a melhor solução atendendo os objetivos propostos de custos e atendimento de pressões. Tal dificuldade se deve a região com grande quantidade de montanhas propiciando grandes variações topográficas.

Recomendam-se futuros estudos com foco em aplicação de métodos de otimização a redes reais de porte médio ou grande para se chegar a melhor eficiência hídrica e energética, especificamente em regiões montanhosas como é o caso deste estudo.

\section{Agradecimentos}

Os autores agradecem ao projeto REDECOPE Finep - MCT (Ref. 0983/10) Ministério da Ciência e Tecnologia intitulado "Desenvolvimento de tecnologias eficientes para a gestão hidro energética em sistemas de abastecimento de água" e Programa Pesquisador Mineiro da FAPEMIG pelo PPM - 00755-16.

\section{Referências}

ABNT (2017). NBR 12.218: Projeto de rede de distribuição de água para abastecimento público. 
Antonowicz, A. et al. (2018). Use of EPANET solver to manage water distribution in Smart City. (2018, February 6). [Paper Presentation]. The First Conference of the International Water Association IWA for Young Scientist in Poland "Water, Wastewater and Energy in Smart Cities", Poland. https://www.e3s-conferences.org/articles/e3sconf/abs/2018/05/e3sconf_iwa2018_01016/e3sconf_iwa2018_01016.html

Farina, G. et al . (2013). Using EPANET for modelling water distribution systems with users along the pipes. Civil Engineering and Enviromental Systems, 31(1), 36-50. https://doi.org/10.1080/10286608.2013.820279

Goulart, T. D. C. (2015) Estudos de Aprimoramento de Algoritmo de Calibração e Aplicação em Rede de Distribuição de Água de Cambuí (MG). Dissertação de Mestrado. https://repositorio.unifei.edu.br/xmlui/handle/123456789/147

Kumar, M. et al. (2014). Pipe Networking For Water Distribution Using EPANET. Global Journal Of Enginnering Science And Researches, (11), 17-20.

Maruf, M. et al. (2015). Water Distribution System Modeling by Using EPANET Software. (2015, November 15). Paper Presentation. International Conference on Recent Innovation in Civl Engineering for Sustainable Development, Bangladesh. http://103.82.172.44:8080/xmlui/handle/123456789/779

Mohammad, M. M. et al. (2015). Water Distribution System Modeling by Using EPANET Software. (2015, November 15). Paper Presentation. International Conference on Recent Innovation in Civl Engineering for Sustainable Development, Bangladesh. http://103.82.172.44:8080/xmlui/handle/123456789/779

Nallanathel, M. et al. (2018). Water distribution network design using EPANET a case study. International Journal of Pure and Applied Mathematics, 119(17), 1165-1172. http://www.acadpubl.eu/hub

Ramana, G. V. et al. (2015). Network Analysis of Water Distribution System in Rural Areas using EPANET. Procedia Engineering, 119, 496-505. https://doi.org/10.1016/j.proeng.2015.08.875

Reis, J. M. (2017). Eficiência energética no sistema de abastecimento de água de Conceição do Araguaia - PA. Monografia de Conclusão de Curso, Faculdade Metropolitana de Anápolis. https://www.faculdadefama.edu.br/wp-content/uploads/2017/07/Efici\%C3\%AAncia-energ\%C3\%A9tica-no-sistema-deabastecimento-de-\%C3\%A1gua-em-Concei\%C3\%A7\%C3\%A3o-do-Araguaia-PA.pdf

Santos, J. (2016) Estudo de Modelo de Calibração para ajuste de parâmetros de rugosidade, demanda e vazamento visando eficiência hidrica e energética em redes de abastecimento de água. Estudo de caso em São Lourenço - MG. Dissertação de Mestrado. https://repositorio.unifei.edu.br/jspui/handle/123456789/500

Saqib, N. et al. (2017). A Multiscale Approach to Leak Detection and Localization in Water Pipeline Network. Water Resources Management, 31(12), 38293842. https://doi.org/10.1007/s11269-017-1709-3

Silva, F. G. B. (2003). Estudos de calibração de redes de distribuição de água através de algoritmos genéticos. Tese de Doutorado. http://www.teses.usp.br/teses/disponiveis/18/18138/tde-23112016-163209/pt-br.php

SINAPI. (2018). Sistema Nacional de Índices e Preços para Construção. http://www.caixa.gov.br/poder-publico/apoio-poderpublico/sinapi/Paginas/default.aspx

Suse, R. et al. (2014). Simulação da Qualidade de Água em Redes de Distribuição Empregando o EPANET-MSX. Revista DAE, (197), 10. https://doi.org/10.4322/dae.2014.126

Tack, H. H. et al. (2016). Gestão do Projeto Temarp - Técnicas Experimentais e de Modelagem Aplicadas à Redução de Perdas em sistemas de Abastecimento Público de Água. (2016, March 21). Paper Presentation. III Simpósio Internacional De Gestão De Projetos (III SINGEP), http://hdl.handle.net/123456789/1022

Uber, J. G. et al. (2018). Let's Get Moving and Write Software: An Open Source Project for EPANET. (2018). Journal of Water Resources Planning and Management, 144(4). https://doi.org/10.1061/(ASCE)WR.1943-5452.0000918

Vieira, L. V. A. (2013). Aplicação do modelo de simulação EPANET 2.0 ao estudo das pressões e cloro residual do sistema de abastecimento de água de Angra do Heroísmo. Dissertação de Mestrado. https://repositorio.uac.pt/handle/10400.3/2799 